\title{
Thromboembolic Risks of Non-Factor Replacement Therapies in Hemophilia
}

\author{
A. Tiede \\ Hematology, Hemostasis, Oncology and Stem Cell Transplantation, Hannover Medical School, Hannover, Germany
}

Impressive advances in the field of hemophilia have been reported during the recent congress of the International Society on Thrombosis and Haemostasis (ISTH) in Berlin in July 2017. Two of the major oral abstract presentations presented studies in the field of hemophilia that appeared the same day as full publications in the New England Journal of Medicine (1, 2), reinforcing the importance of hemophilia as a driver of innovation in hemostasis.

The new non-factor replacement therapies promise to overcome two important shortcomings of traditional factor replacement therapy: (i) the need of frequent intravenous administrations; and (ii) the risk of transient or permanent formation of neutralizing antibodies, so-called inhibitors $(3,4)$.

\section{Hemophilia with inhibitors: state of the art}

The mainstay of treatment in congenital hemophilia with inhibitors (CHWI) is immune tolerance induction (ITI), aiming at eradicating inhibitors and inducing tolerance to factor VIII or IX (5). This treatment involves repeated administration of clotting factor, twice daily in the most intense protocols (6). Placement of central venous lines is usually required. The treatment is successful

\footnotetext{
Correspondence to:

Andreas Tiede, MD, PhD

Hematology, Hemostasis, Oncology and Stem Cell Transplantation

Hannover Medical School

Carl-Neuberg-Str. 1,

30175 Hannover, Germany

Tel. +495115324147

Fax +495115324146

Email tiede.andreas@mh-hannover.de
}

Hämostaseologie 2017; 37: 307-310

received: September 19, 2017

accepted after minor revision: September 21, 2017 in 60 to $80 \%$ after several months of treatment (7), and is often hampered by problems with venous access, including line infection and thrombosis (8). Until tolerance is achieved, bleeds are difficult to prevent and difficult to control, resulting in higher risks of morbidity, mortality, and disability (9-12). FVIII bypassing agents (BPA), including activated prothrombin complex concentrate (APCC) and recombinant activated factor VII (rFVIIa) can restore hemostasis in the absence of factor VIII (FVIII) or IX (FIX) and effectively stop acute bleeds in most patients (13). However, the ability of those BPA to prevent bleeds in a prophylactic setting is limited, partly because of their short half-life (14-16).

These issues set the scene for the development of the novel non-factor replacement therapies (17). Representing diverse mechanisms of action, they have in common (i) efficacy independent of the presence of inhibitors, (ii) long half-life, (iii) subcutaneous administration, and (iv) reduced or absent risk of anti-drug antibodies. However, first reports on thromboembolic events remind us that groundbreaking innovation may not only offer unprecedented success but also unexpected pitfalls and risks.

\section{Emicizumab: FVIIla-mimetic bispecific antibody}

Developed under the name of ACE 910, emicizumab is a humanized bispecific antibody targeting FIX/FIXa and factor X (FX) to support the activation of FX. Emicizumab will replace FVIIIa in hemophilia A with or without inhibitors. It is not useful in hemophilia B.

The clinical study results published so far underline the great potential of the drug $(1,18)$. The HAVEN 1 study presented on ISTH 2017 was an open-label, randomized controlled study of 109 adult and adolescent patients with hemophilia A and in- hibitors (1). Patients previously using ondemand BPA were randomized to prophylaxis with emicizumab (arm A, $1.5 \mathrm{mg} / \mathrm{kg}$ weekly, $n=35$ ) or continued on-demand BPA ( $\operatorname{arm} B, n=18)$. During the study period of 24 weeks, the number of treated bleeds was $87 \%$ lower in patients on emicizumab (mean annualized bleeding rate [ABR] 2.9; 95\% confidence interval 1.7-5.0) compared to on-demand BPA (23.3; 12.3-43.9). Patients previously on prophylactic BPA switched to emicizumab without randomization (arm C) and experienced a $79 \%$ reduction in bleeds (mean ABR $3.3 ; 1.3-8.1)$ compared to the historic control period (15.7; 11.1-22.3).

The drug was generally well tolerated. The most frequent adverse event was injection site reaction in $15 \%$ of patients. Four thromboembolic events were reported in the primary analysis. This was amended to a total of 5 events in 5 patients during the ISTH conference oral presentation by Oldenburg et al. (results update as of April 2017, [19]): 3 cases of thrombotic microangiopathy (TMA), and 2 venous thromboembolic events, including a sinus vein thrombosis, and a superficial thrombophlebitis. One patient died in the context of rectal bleeding that could no longer be treated with APCC due to the TMA.

\section{Emicizumab: a closer look at thromboembolic events}

In HAVEN 1, all thromboembolic events occurred during episodes of treatment with APPC for breakthrough bleeding. The risk appeared to be dose-related: the 5 events occurred during 5 out of 8 treatment episodes of APCC $>100 \mathrm{U} / \mathrm{kg}$ per day for $>24 \mathrm{~h}$. In contrast, no events occurred with lower doses of APCC $(\leq 100 \mathrm{U} / \mathrm{kg})$ or shorter treatment durations $(\leq 24 \mathrm{~h})$, or when rFVIIa was used for breakthrough bleeding ( $\triangleright$ Fig. 1). 


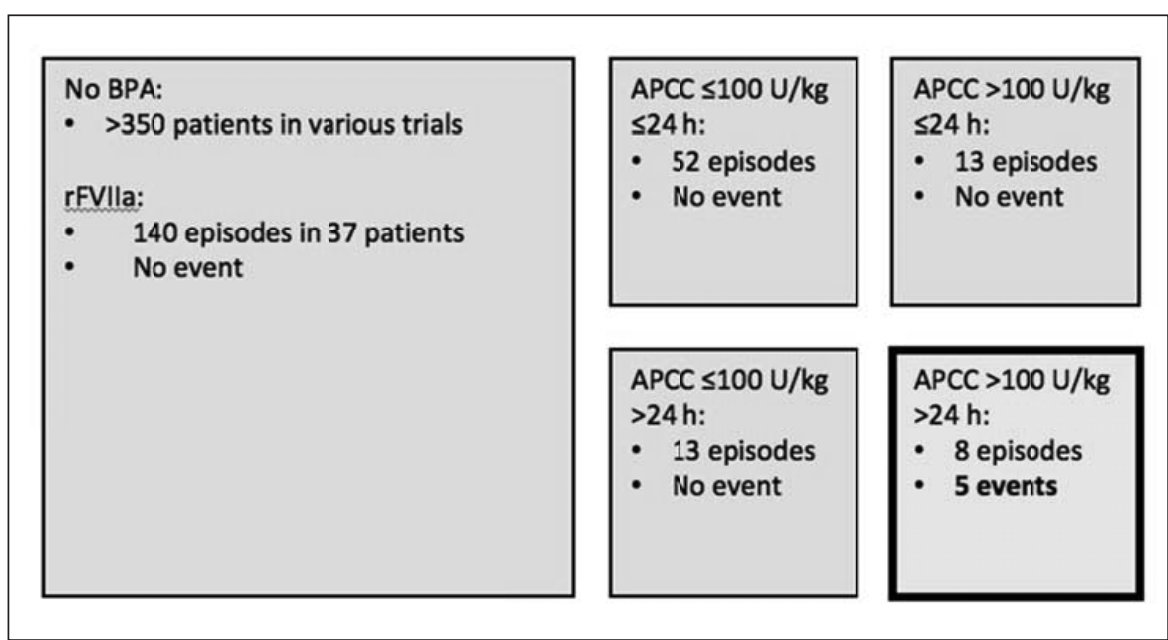

Fig. 1 Thromboembolic events observed with emicizumab.

Even though thrombosis was occasionally observed with APCC (20) and rFVIIa $(21,22)$, TMA is a truly novel form of thromboembolic complication that was seen neither with APCC or rFVIIa alone, nor with emicizumab alone in $>350$ patients enrolled altogether in the ongoing study program.

It will be important to better understand the pathomechanism of thromboembolic events during treatment with emicizumab. The drug's affinity to FIX/IXa and FX/Xa is lower in solution than that reported for FVIIIa on phospholipid membranes (23). The $K_{\mathrm{D}}$-based simulations predict that with therapeutic plasma concentrations of emicizumab, the majority of FIX and FX exist as monomers, followed by emicizumabFIX and emicizumab-FX dimers, and only a very small fraction as ternary FIX-emicizumab-FX complexes. However, these measurements have not been performed on phosphatidylserine-exposed phospholipid membranes or in the presence of APCC. It is tempting to speculate that active phospholipid membranes and/or increased concentrations of (pro-)enzyme FIX(a) or substrate FX may increase the drug's ability to support FXa generation and result in excessive thrombin formation.

For the time being, the manufacturer recommended to avoid the combination of emicizumab with APCC and, if APCC was needed, to use it at the lowest dose possible. It remains to be seen whether APCC can be avoided in all CHWI patients receiving emicizumab, given the established experience that some patients respond clinically differently to the BPA and may not be sufficiently treated with rFVIIa.

\section{Fitusiran: antithrombin silencing}

Fitusiran is a small interfering ribonucleic acid (siRNA), coupled to $\mathrm{N}$-acetylgalactosamine (GalNAc). GalNAc-siRNA conjugates can be administered subcutaneously and will be targeted to hepatocytes through uptake by the asialoglycoprotein receptor. Inside the cell, it will turn off the expression of antithrombin (SERPINC1) mRNA to achieve stable and consistent reduction in plasma antithrombin activity to about $20 \%$ of baseline (2).

Low antithrombin, in the context of congenital deficiency, has been reported to increase thrombin levels (24). Occasionally, hemophilia patients with a less severe bleeding phenotype have been found to carry inherited thrombophilic 'risk' factors including antithrombin deficiency (25). Heterozygous antithrombin deficiency also mitigates the phenotype of FVIII deficiency in the hemophilia A mouse model (26). These observations formed the rational for the development of antithrombin silencing by RNA interference in hemophilia.

The phase 1 dose-escalation study enrolled 4 healthy individuals and 25 patients with moderate or severe hemophilia A or B (26). According to the June 2017 data update of the phase 2 open-label extension study presented during ISTH (27), 33 patients with hemophilia A or B, with or without inhibitors, have been treated for a median of 11 months. The median ABR for non-inhibitor patients was $1.7(\mathrm{n}=19)$, and for inhibitor patients $0(n=14)$. This compared with a historic ABR of 2 (non-inhibitor patients previously on prophylaxis, $\mathrm{n}=7$ ), ABR of 12 (non-inhibitor patients previously treated on demand, $n=12$ ), and $A B R$ of 38 (patients with inhibitors, $n=14$ ), suggesting that fitusiran can prevent bleeds in most patients with and without inhibitors.

The drug was generally well tolerated. Injection-site reactions were seen in $18 \%$ of patients; abdominal pain, diarrhea, and headache occurred in $9 \%$ each. Asymptomatic alanine transferase (ALT) increases $\geq 3 \mathrm{x}$ upper limit of normal occurred in 11 patients (33\%), all of which were hepatitis $\mathrm{C}$ antibody positive.

Of note, breakthrough bleeds in non-inhibitor patients were managed with low doses of FVIII (18 bleeds; mean 17 [range 5-31] IU/kg for a mean of 1.1, [range 1-2] doses) or FIX (7 bleeds; 18 [9-27] IU/kg for 3.9 [1-8] doses). All patients used less than or same amount of factor per bleed as prior to fitusiran. In inhibitor patients, APCC (56 bleeds; 27 [14-37] U/kg for 1.5 [1-3] doses) and rFVIIa (3 bleeds; 56 [37-62] $\mu \mathrm{g} / \mathrm{kg}$ for 2.7 [2-3] doses) were also dosed less intensely in most patients. No thromboembolic events were reported in these patients.

\section{Fitusiran: first thromboembolic event}

Most recently, a first thromboembolic event with fitusiran was reported (28). According to the study sponsor's press release, this was a patient, who treated an exerciseinduced hip pain with three doses of FVIII (31-46IU/kg). On the last day of dosing, he developed severe headache, was diagnosed with subarachnoid hemorrhage on CT scan, and received further treatment with FVIII. Over a 14-day hospitalization his condition worsened and he died from 
cerebral edema. Post-hoc review of the initial CT scan, however, confirmed that the initiating event was a sinus vein thrombosis rather than subarachnoid hemorrhage. The sponsor suspended fitusiran dosing in all patients currently enrolled in the trial program.

\section{Comment: where do we go from here?}

Although both drugs hold great promise as a potential treatment for patients with and without inhibitors, the thromboembolic events observed during the trial programs raise concerns about their safety. We must concede that thrombosis used to be a rare event in the hemophilia population, even in elderly patients and during treatment with BPA. Of note, the thromboembolic events seen in emicizumab and fitusiran trial programs were mostly atypical in nature, including sinus vein thrombosis and TMA. Deep vein thrombosis, the most frequent form of venous thromboembolism in the general population, has so far not been observed. This suggests a distinct pathophysiology of thromboembolism in patients treated with the non-factor replacement therapies that needs to be explored carefully.

First, more basic research is needed to understand mechanisms of action. How do emicizumab and APCC interact with each other? How can hemostasis be limited to the site of injury in the presence of emicizumab? What happens if emicizumab dosing is increased, either intentionally or accidentally?

Second, we need dose finding studies for BPA and factor concentrates in patients treated with emicizumab or fitusiran. Thrombin generation experiments and occasional observations in patients treated in the trial programs indicate that lower doses and shorter treatment durations of BPA or FVIII may be adequate to restore hemostasis in the event of breakthrough bleeds. However, this needs to be studied more carefully at the clinical level. For the time being, the combination of emicizumab with APCC $>100 \mathrm{U} / \mathrm{kg}$ for $>1$ day appears to be clearly contraindicated. However, it may not be feasible to abstain from APCC use in all inhibitor patients. Surgery and major bleeds may require the use of BPA for prolonged periods of time, posing significant uncertainties on how to dose APCC, but also rFVIIa, FVIII and FIX. Laboratory monitoring of coagulation activation markers or fragmented red blood cells (schistocytes) could potentially help to recognize thromboembolic risks earlier, but this needs systematic investigation in the ongoing and forthcoming studies.

Third, the development of antidotes should be considered. An antidote against emicizumab is currently not available, but might be warranted in certain clinical scenarios, very much like the antidotes against the direct oral anticoagulants. Antithrombin lowering by fitusiran can theoretically be reversed by infusion of antithrombin concentrate. However, the clinical scenario where this might be useful must be carefully considered and experience should be systematically collected in studies and registries.

\section{Conclusion}

The non-factor replacement therapies are groundbreaking developments addressing a major unmet medical need. They have the potential to revolutionize hemophilia treatment, both for patients with and without inhibitors. However, in the field of hemostasis and thrombosis we had to realize often that hemostasis and thrombosis are two sides of the coin that cannot be easily separated. Bleeding has always been the major concern of antithrombotic therapies; and thrombosis turns out to be the major issue with the new hemophilia treatments. Again, we are facing how difficult it is to keep the balance.

\section{Acknowledgments}

The author acknowledges the input of Prof. Dr. Johannes Oldenburg, communicating contents of the ISTH presentation of emicizumab data. Prof. Dr. Rüdiger E. Scharf, EIC Hämostaseologie - Progress in Haemostasis, is acknowledged for critical review and helpful editorial comments to this article.
Disclosure of conflict of interest (COI):

AT reports grants and personal fees for lectures and consultancy from Alnylam, Bayer, Biogen Idec, Biotest, Boehringer Ingelheim, CSL Behring, Leo Pharma, Novo Nordisk, Octapharma, Pfizer, Roche, Shire, and SOBI.

\section{References}

1 Oldenburg J, Mahlangu JN, Kim B, Schmitt C, Callaghan MU, Young G, Santagostino E, Kruse-Jarres R, Negrier C, Kessler C, Valente N, Asikanius E, Levy GG, Windyga J, Shima M. Emicizumab Prophylaxis in Hemophilia A with Inhibitors. N Engl J Med 2017; 377: 809-818.

2 Pasi KJ, Rangarajan S, Georgiev P, Mant T, Creagh MD, Lissitchkov T, Bevan D, Austin S, Hay CR, Hegemann I, Kazmi R, Chowdary P, GerchevaKyuchukova L, Mamonov V, Timofeeva M, Soh CH, Garg P, Vaishnaw A, Akinc A, Sorensen B, Ragni MV. Targeting of Antithrombin in Hemophilia A or B with RNAi Therapy. N Engl J Med 2017; 377: 819-828.

3 Astermark J. FVIII inhibitors: pathogenesis and avoidance. Blood 2015; 125: 2045-2051.

4 Scharf RE. Hot topic: Alloantibodies to therapeutic FVIII and the use of emicizumab in hemophilia A. Hämostaseologie 2016; 36: 195-198.

5 Holstein K, Batorova A, Carvalho M, Fijnvandraat K, Holme P, Kavakli K, Lambert T, Rocino A, Jimenez-Yuste V, Astermark J, European Haemophilia Therapy Strategy B. Current view and outcome of ITI therapy - A change over time? Thromb Res 2016; 148: 38-44.

6 Oldenburg J, Schwaab R, Brackmann HH. Induction of immune tolerance in haemophilia $\mathrm{A}$ inhibitor patients by the ,Bonn Protocol': predictive parameter for therapy duration and outcome. Vox Sang 1999; 77 (Suppl. 1): 49-54.

7 Hay CR, DiMichele DM. The principal results of the International Immune Tolerance Study: a randomized dose comparison. Blood 2012; 119: 1335-1344.

8 Rodriguez V, Mancuso ME, Warad D, Hay CR, DiMichele DM, Valentino L, Kenet G, Kulkarni R. Central venous access device (CVAD) complications in Haemophilia with inhibitors undergoing immune tolerance induction: Lessons from the international immune tolerance study. Haemophilia 2015; 21: e369-e374.

9 Morfini M, Haya S, Tagariello G, Pollmann H, Quintana M, Siegmund B, Stieltjes N, Dolan G, Tusell J. European study on orthopaedic status of haemophilia patients with inhibitors. Haemophilia 2007; 13: 606-612.

10 Gringeri A, Mantovani LG, Scalone L, Mannucci PM, Group CS. Cost of care and quality of life for patients with hemophilia complicated by inhibitors: the COCIS Study Group. Blood 2003; 102: 2358-2363.

11 Scalone L, Mantovani LG, Mannucci PM, Gringeri A, Investigators CS. Quality of life is associated to 
the orthopaedic status in haemophilic patients with inhibitors. Haemophilia 2006; 12: 154-162.

12 Holstein K, von Mackensen S, Bokemeyer C, Langer $\mathrm{F}$. The impact of bleeding disorders on the socioeconomic status of adult patients. Results of a comparative single centre cohort study. Hämostaseologie 2017; 99

13 Astermark J, Donfield SM, DiMichele DM, Gringeri A, Gilbert SA, Waters J, Berntorp E, Group FS. A randomized comparison of bypassing agents in hemophilia complicated by an inhibitor: the FEIBA NovoSeven Comparative (FENOC) Study. Blood 2007; 109: 546-551.

14 Antunes SV, Tangada S, Stasyshyn O, Mamonov V, Phillips J, Guzman-Becerra N, Grigorian A, Ewenstein B, Wong WY. Randomized comparison of prophylaxis and on-demand regimens with FEIBA NF in the treatment of haemophilia A and B with inhibitors. Haemophilia 2014; 20: 65-72.

15 Leissinger C, Gringeri A, Antmen B, Berntorp E Biasoli C, Carpenter S, Cortesi P, Jo H, Kavakli K, Lassila R, Morfini M, Negrier C, Rocino A, Schramm W, Serban M, Uscatescu MV, Windyga J, Zulfikar B, Mantovani L. Anti-inhibitor coagulant complex prophylaxis in hemophilia with in hibitors. N Engl J Med 2011; 365: 1684-1692.

16 Konkle BA, Ebbesen LS, Erhardtsen E, Bianco RP, Lissitchkov T, Rusen L, Serban MA. Randomized, prospective clinical trial of recombinant factor
VIIa for secondary prophylaxis in hemophilia patients with inhibitors. J Thromb Haemost 2007; 5 : 1904-1913.

17 Klamroth R. A new era of treatment for patients with haemophilia A? Hämostaseologie 2017; 37: 216-218.

18 Shima M, Hanabusa H, Taki M, Matsushita T, Sato T, Fukutake K, Fukazawa N, Yoneyama K, Yoshida H, Nogami K. Factor VIII-Mimetic Function of Humanized Bispecific Antibody in Hemophilia A. N Engl J Med 2016; 374: 2044-2053.

19 Roche press release of 26th June 2017. http://www. roche.com/media/store/releases/medcor-2017-06-26.htm. Accessed on 21st September 2017.

20 Luu H, Ewenstein B. FEIBA safety profile in multiple modes of clinical and home-therapy application. Haemophilia 2004; 10 (Suppl. 2): 10-16.

21 Neufeld EJ, Negrier C, Arkhammar P, Benchikh el Fegoun S, Simonsen MD, Rosholm A, Seremetis S. Safety update on the use of recombinant activated factor VII in approved indications. Blood Rev 2015; 29 (Suppl. 1): S34-S41.

22 Tiede A, Amano K, Ma A, Arkhammar P, El Fegoun SB, Rosholm A, Seremetis S, Baudo F. The use of recombinant activated factor VII in patients with acquired haemophilia. Blood Rev 2015; 29 (Suppl. 1): S19-S25.
23 Kitazawa T, Esaki K, Tachibana T, Ishii S, Soeda T, Muto A, Kawabe Y, Igawa T, Tsunoda H, Nogami K, Shima M, Hattori K. Factor VIIIa-mimetic cofactor activity of a bispecific antibody to factors IX/IXa and $\mathrm{X} / \mathrm{Xa}$, emicizumab, depends on its ability to bridge the antigens. Thromb Haemost 2017; 117: 1348-1357.

24 Alhenc-Gelas M, Canonico M, Picard V. Influence of natural SERPINC1 mutations on ex vivo thrombin generation. J Thromb Haemost 2010; 8: 845-848.

25 Shetty S, Vora S, Kulkarni B, Mota L, Vijapurkar M, Quadros L, Ghosh K. Contribution of natural anticoagulant and fibrinolytic factors in modulating the clinical severity of haemophilia patients. $\mathrm{Br}$ J Haematol 2007; 138: 541-544.

26 Bolliger D, Szlam F, Suzuki N, Matsushita T, Tanaka KA. Heterozygous antithrombin deficiency improves in vivo haemostasis in factor VIII-deficient mice. Thromb Haemost 2010; 103: 1233-1238.

27 Alnylam press release of 10th July 2017. http://investors.alnylam.com/releasedetail.cfm?ReleaseID $=1032569$. Accessed on 21st September 2017

28 Alnylam press release of 7 th September 2017. http://investors.alnylam.com/releasedetail.cfm?ReleaseID=1039464. Accessed on 21st September 2017. 